\title{
NFATC3-PLA2G15 Fusion Transcript Identified by RNA Sequencing Promotes Tumor Invasion and Proliferation in Colorectal Cancer Cell Lines
}

\author{
Jee-Eun Jang, $\mathrm{PhD}^{1}$ \\ Hwang-Phill Kim, PhD',2 \\ Sae-Won Han, MD, PhD ${ }^{1,3}$ \\ Hoon Jang, $\mathrm{PhD}^{4}$ \\ Si-Hyun Lee, MS ${ }^{1}$ \\ Sang-Hyun Song, $\mathrm{PhD}^{1}$ \\ Duhee Bang, PhD ${ }^{4}$ \\ Tae-You Kim, MD, PhD',2,3
}

${ }^{1}$ Cancer Research Institute, Seoul National University College of Medicine, Seoul, ${ }^{2}$ Department of Molecular Medicine and Biopharmaceutical Sciences, Graduate School of Convergence Science and Technology, Seoul National University College of Medicine, Seoul, ${ }^{3}$ Department of Internal Medicine, Seoul National University Hospital, Seoul, ${ }^{4}$ Department of Chemistry, College of Science, Yonsei University, Seoul, Korea

Correspondence: Sae-Won Han, MD, PhD Department of Internal Medicine, Seoul National University Hospital, 101 Daehak-ro, Jongno-gu, Seoul 03080, Korea Tel: 82-2-2072-0242

Fax: 82-2-762-9662

E-mail: saewon1@snu.ac.kr

Co-correspondence: Tae-You Kim, MD, PhD Department of Internal Medicine, Seoul National University Hospital, 101 Daehak-ro, Jongno-gu, Seoul 03080, Korea Tel: 82-2-2072-3943

Fax: 82-2-762-9662

E-mail: kimty@snu.ac.kr

Received February 9, 2018

Accepted May 28, 2018

Published Online June 14, 2018

*Jee-Eun Jang and Hwang-Phill Kim contributed equally to this work.

\section{Purpose}

This study was designed to identify novel fusion transcripts (FTs) and their functional significance in colorectal cancer (CRC) lines.

\section{Materials and Methods}

We performed paired-end RNA sequencing of 28 CRC cell lines. FT candidates were identified using TopHat-fusion, ChimeraScan, and FusionMap tools and further experimental validation was conducted through reverse transcription-polymerase chain reaction and Sanger sequencing. FT was depleted in human CRC line and the effects on cell proliferation, cell migration, and cell invasion were analyzed.

\section{Results}

One thousand three hundred eighty FT candidates were detected through bioinformatics filtering. We selected six candidate FTs, including four inter-chromosomal and two intrachromosomal FTs and each FT was found in at least one of the 28 cell lines. Moreover, when we tested 19 pairs of CRC tumor and adjacent normal tissue samples, NFATC3PLA2G15 FT was found in two. Knockdown of NFATC3-PLA2G15 using siRNA reduced mRNA expression of epithelial-mesenchymal transition (EMT) markers such as vimentin, twist, and fibronectin and increased mesenchymal-epithelial transition markers of E-cadherin, claudin-1, and FOXC2 in colo-320 cell line harboring NFATC3-PLA2G15 FT. The NFATC3-PLA2G15 knockdown also inhibited invasion, colony formation capacity, and cell proliferation.

\section{Conclusion}

These results suggest that that NFATC3-PLA2G15 FTs may contribute to tumor progression by enhancing invasion by EMT and proliferation.

Key words

Colorectal neoplasms, Fusion transcript, RNA-sequencing, NFATC3, PLA2G15 


\section{Introduction}

Colorectal cancer $(\mathrm{CRC})$ is the third most common cancer diagnosis, and is the leading cause of cancer-related death worldwide [1]. However, because existing therapies can be toxic, more specific therapeutic regimens, such as targeted agents, have been sought to improve the outcomes and quality of life of patients with CRC [2]. To do so, many studies are underway to discover new targets for targeted therapy through genome analysis.

Fusion transcripts (FTs) were recently reported to produce feasible drug targets, which implies that detection of FTs could be a plausible diagnostic and / or treatment strategy for some malignancies [3-6]. Characterization of specific FTs in cancers has led to the identification of several successful therapeutic targets, such as BCR-ABL1 [7]. FTs are a class of genetic alterations that result from chromosomal rearrangements [8]. FTs that result in tumorigenesis are potential drug targets in several cancers [9]. Many tyrosine kinase rearrangements occur in epithelial cancers, including anaplastic lymphoma kinase (ALK), proto-oncogene tyrosine-protein kinase ROS (ROS1), ret proto-oncogene (RET), neurotrophic tyrosine kinase, receptor, type 1 (NTRK1), fibroblast growth factor receptors (FGFR), AXL receptor tyrosine kinase (AXL), and platelet-derived growth factor receptor, alpha polypeptide (PDGFRA) [10]. Among these tyrosine kinase fusions transcripts, the echinoderm microtubule-associated proteinlike 4 ALK (EML4-ALK) fusion, which was discovered in non-small-cell lung cancer (NSCLC) [11], has been targeted by crizotinib for NSCLCs that harbor the EML4-ALK fusion gene [12].

Of the many FTs that have been discovered in CRC thus far, almost all, including VTI1A-TCF7L2 [13], PTPRK-RSPO3 [14], and TCF7L2-RP11-57H14.3 [15], affect the Wnt signaling pathway. Many FTs have been discovered in CRC, including AKAP13-PDE8A, COMMD10-AP3S1, SLC39A14TSPAN15, NCOA3-SPINT1, GRIN2B-CYP4F3, PRMT1-FLT$3 L G$, and $V N N 1-A B[16,17]$. However, with the exception of VTL1A-TCF7L2 and PTPRK-RSPO3, their functions have not been studied. As with other FTs in diverse tumors, the identification of FTs in CRC could provide new insights about this disease.

Here, we conducted paired-end RNA sequencing to discover novel FTs in CRC cell lines. NFATC3-PLA2G15 FT was also detected in CRC tumor tissue. We conducted experiments aimed at a functional study of the NFATC3-PLA2G15 FT. We observed that NFATC3-PLA2G15 FT was involved in the regulation of invasion, anchorage-independent features, and cell proliferation in CRC cell lines.

\section{Materials and Methods}

\section{Cell lines and culture}

Human CRC cell lines (colo-201, colo-205, colo-320, HCT15, HCT-116, HT-29, Lovo, LS174T, SNU-61, SNU-70, SNU81, SNU-175, SNU-254, SNU-283, SNU-407, SNU-977, SNU1033, SNU-1181, SNU-1235, SNU-1411, SNU-1544, SNU1684, SNU-C1, SNU-C2A, SNU-C4, SNU-C5, SW-403, and SW-480) were purchased from the Korean Cell Line Bank [18]. All cell lines were cultured in RPMI1640 (Hyclone Laboratories, Ind., Logan, UT), supplemented with $10 \%$ fetal bovine serum, at $37^{\circ} \mathrm{C}$ in humidified $5 \% \mathrm{CO}_{2}$.

\section{Paired-end RNA sequencing and fusion analysis}

Total RNA was extracted from each cell line using an RNeasy mini kit (Qiagen, Valencia, CA). The mRNA was isolated from $1 \mu \mathrm{g}$ of total RNA using the NEBNext Poly mRNA Magnetic Isolation Module. The cDNA library was prepared from mRNA using the NEBNext Ultra RNA Library Prep Kit for Illumina (NEB) and sequenced using the Illumina HiSeq 2500 platform $(2 \times 150 \mathrm{bp})$.

Next-generation sequencing reads were analyzed with three fusion detection tools including TopHat-fusion, ChimeraScan, and FusionMap. TopHat-fusion (v.2.0.9) analysis was performed using Bowtie (v.1.0.1) with fusionsearch option and default parameters. ChimeraScan (v.0.4.5) and FusionMap (2013-07-30) were also performed using default parameters. All of the programs used hg19 (GRCh37) and RefGene as reference genome and gene model, respectively. From all of the results analyzed using the three tools, we obtained fusions with four or more supporting reads for further analysis.

\section{Human colon cancer tissues}

We obtained 19 human colon adenocarcinoma tissues and matched normal tissues from the tissue bank of Seoul National University Hospital (Seoul, Korea). After resection, the tissues were immediately frozen in liquid nitrogen and stored until needed.

\section{Reverse transcriptase-polymerase chain reaction and quantitative real-time polymerase chain reaction}

Total RNA was extracted from each cell line for transcriptome sequencing using an RNeasy mini kit (Qiagen) according to the manufacturer's instructions. The cDNA was synthesized from total RNA ( $1 \mu \mathrm{g})$ using ImProm-II reverse transcriptase (Promega, Madison, WI) and amplified by reverse 
transcriptase-polymerase chain reaction (RT-PCR) using AmpliTaq Gold DNA polymerase (Applied Biosystems, Foster City, CA) with buffer supplied by the manufacturer and gene/fusion junction-specific primers. For quantitative realtime RT-PCR (qRT-PCR), cDNA was amplified using Premix Ex Taq (TaKaRa, Shiga, Japan) with SYBR Green I (Molecular Probes, Eugene, OR) using a StepOnePlus Real-Time PCR system (Applied Biosystems).

\section{Sanger sequencing}

Sanger-sequencing reactions were conducted in both forward and reverse reactions. NFATC3-PLA2G15 FT junction was amplified using the following forward (F): GTGTGATCCAGCGTCATTT and reverse (R): AGTAGGGCCCGTTTTCATTT primers. Sequencing was carried out by Macrogen Inc. (Seoul, Korea).

\section{IGV data analysis}

The IGV program (version IGV_2.3.32) was downloaded from https://www.broadinstitute.org/igv/. After loading the BAM file from the RNA-sequencing raw data, the $3^{\prime}$ position of NFATC3 and the $5^{\prime}$ position of PLA2G15 were analyzed.

\section{Small interfering RNA knockdown}

The siRNAs used in this study, including the control siRNA (si-CONTROL) with scrambled sequences, were synthesized by Genolution Pharmaceuticals, Inc. (Seoul, Korea). The siRNA sequences used against the NFATC3-PLA2G15 FT were 5'-GAUGAUGUCCCUGGUGAUUUU-3' (sense) and 5'-AAUCACCAGGGACAUCAUCUU-3' (antisense), which targeted the FT breakpoint. Each cell line was treated with siRNA (50 nM) for 48 hours. Lipofectamine 2000 (Invitrogen, Carlsbad, CA) was used for transfection, according to manufacturer's instructions.

\section{Soft agar assay}

Noble agar (Difco, HR, India), which was prepared by combining $1.5 \mathrm{~mL}$ of $0.5 \%$ agar in RPMI1640 with $20 \%$ fetal bovine serum (FBS), was solidified in the bottom of each well of a 6-well plate. On the next day, si_NFATC3-PLA2G15 fusion and control transfected cells $\left(1 \times 10^{4}\right)$ in RPMI1640 with $20 \%$ FBS media were mixed with $0.4 \%$ agarose and overlaid onto the bottom agar. The plates were then incubated in a 5\% $\mathrm{CO}_{2}$ incubator at $37^{\circ} \mathrm{C}$. After 3 weeks, colonies were counted under a microscope and numbers of colonies from triplicate wells were averaged. Colonies were imaged using a microscope equipped with a digital camera.

\section{Invasion assay}

$3 \times 10^{5}$ colo- 320 cells were stably transfected with si-control and si-NFATC3-PLA2G15 and $3 \times 10^{4}$ cells were seeded on the Matrigel-coated membrane matrix (BD Biosciences, San Jose, CA) insert well according to manufacturer's instructions after 48 hours. Media containing 20\% FBS was added as a chemoattractant to the wells of the Matrigel invasion chamber for 24 hours. The following day, the cells were fixed for 10 minutes in $3.7 \%$ paraformaldehyde and the insert was washed with phosphate-buffered saline (PBS). Crystal violet $(0.1 \%)$ was added to the insert for 10 minutes and washed twice with PBS, and then with water. A cotton swab was used to remove any non-invading cells and the insert was washed again. The number of invading cells was imaged using a microscope equipped with a digital camera.

\section{Cell proliferation assay}

Colo-320 cells were plated in completed growth media in $60 \mathrm{~mm}$ dishes $\left(7 \times 10^{5}\right.$ cells per dish) and treated with si_NFATC3-PLA2G15 and si_control on the following day. Cell numbers were counted using a hemocytometer for 4 days in a time-dependent manner. Three independent experiments were performed, and the results averaged.

\section{Western blotting}

Cultured cells were washed with ice-cold PBS and lysed with lysis buffer (50 mM Tris- $\mathrm{HCl}$ [pH 7.5], 1\% NP-40, 0.1\% sodium deoxycholate, $150 \mathrm{mM} \mathrm{NaCl}, 50 \mathrm{mM} \mathrm{NaF}, 1 \mathrm{mM}$ sodium pyrophosphate, $1 \mathrm{mM}$ EDTA, and protease/phosphatase inhibitors). Sodium dodecyl sulfate polyacrylamide gel electrophoresis was used to resolve $20 \mu \mathrm{g}$ of total protein. The resolved proteins were then transferred to nitrocellulose membranes. After blocking with $1 \%$ skim milk and $1 \%$ bovine serum albumin/Tris-buffered saline with Tween 20, the membranes were incubated with primary antibodies at $4^{\circ} \mathrm{C}$ overnight. Antibody against cyclin D were purchased from Cell Signaling Technology (Beverley, MA). Antibodies against vimentin, E-cadherin, cyclin E, and p27 were purchased from Santa Cruz Biotechnology (Santa Cruz, CA). An anti- $\beta$-actin antibody was acquired from Sigma-Aldrich (St. Louis, MO).

\section{Statistics}

Statistical significance of the results was calculated using an unpaired Student's t-test, with a SigmaPlot program. $\mathrm{p}<$ 0.05 was considered significant. 


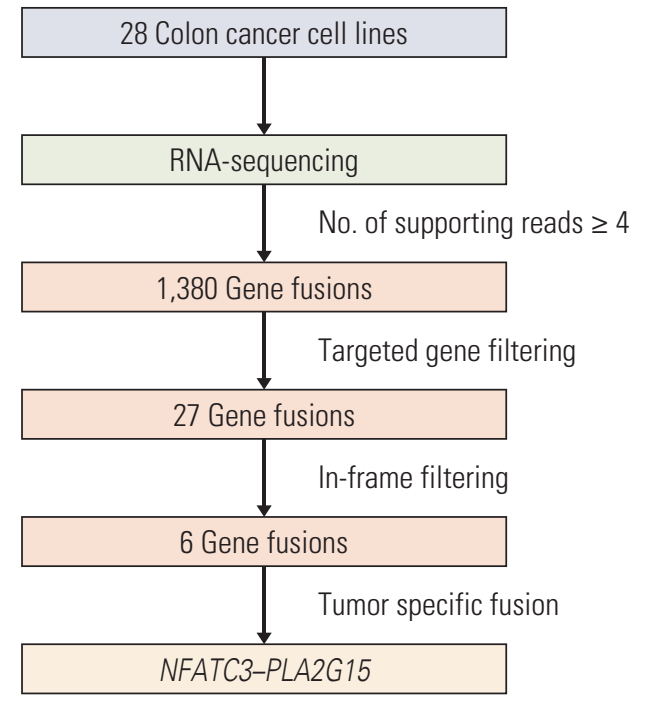

Fig. 1. A schematic of the fusion candidate filtering process.

\section{Ethical statement}

This study protocol was approved by the institutional review board of Seoul National University Hospital (IRB protocol number: H-1305-587-490). Informed consent had been obtained from each patient prior to surgery.

\section{Results}

\section{Paired-end sequencing and identification of FTs}

To discover novel gene fusions in CRC, we performed paired-end RNA sequencing using 28 CRC cell lines. Average depth of RNA-sequencing data was $154 \times$ and median depth was 146×. Statistics of each RNA-sequencing data was indicated in S1 and S2 Table. The list of FT candidates was produced by using the TopHat-fusion, ChimeraScan, and FusionMap tools, for a total of 1,380 candidates with at least four spanning read counts at each of the tools.

We followed a targeted approach to filter the 1,380 gene fusions that contained Wnt signaling pathway-related genes and kinase domains for target gene selection. First, we obtained the list of Wnt signaling pathway-related genes from the Kyoto Encyclopedia of Genes and Genomes (KEGG) pathway because activation of the Wnt signaling pathway is a critical event in the development of CRC. Second, we composed a list of the genes from DAVID that included a kinase domain, which could be a biomarker for a therapeutic target.

In the targeted filtering step, 139 WNT-related genes and 11 genes with kinase domains were selected as FT candidates. After targeted filtering, 27 FTs remained. We removed 21 gene fusions because they did not have in-frame sequences where the two genes were fused (Fig. 1). Finally, four intra-chromosomal FTs (PPP2CA-SKP1, CTNNBIP1-CLSTN1, AKAP13-PDE8A, and NFATC3-PLA2G15) and two interchromosomal FTs (FZD2-RPS24 and KRT8-PKM2) remained (Table 1).

\section{Validation of FTs in cell lines and tumor tissues}

In the validation step, each of six FTs-PPP2CA-SKP1, CTNNBIP1-CLSTN1, AKAP13-PDE8A, NFATC3-PLA2G15, FZD2-RPS24, and KRT8-PKM2-were detected in HCT-15, SNU-81, SW-480, colo-320, HCT-15, and SNU-1235, respectively (Fig. 2A). We next examined the frequency of the FTs in tumor tissues and matched normal tissues. Only NFATC3PLA2G15 FT was detected in two out of 19 (10.5\%) tumor samples, but not in matched normal tissues, which implies that NFATC3-PLA2G15 is a tumor-specific transcript (Fig. 2B). Moreover, age, sex, histology, primary site, microsatellite stable/microsatellite instable status, or tumor stage of the CRC patients did not show a significant correlation (data not shown). The other FTs were not detected in the tumor tissue (data not shown). Therefore, we focused on the NFATC3PLA2G15 FT for further study.

The NFATC3-PLA2G15 FT consisted of exons 1-9 of

Table 1. In-frame fusion transcript candidates in the 28 colon cancer cell lines

\begin{tabular}{lclcc} 
5'-Gene & 5'-Chromosome & 3'-Gene & 3'-Chromosome & Fusion type \\
PPP2CA & 5 & SKP1 & 5 & Intra \\
CTNNBIP1 & 1 & CLSTN1 & 1 & Intra \\
AKAP13 & 15 & $P D E 8 A$ & 15 & Intra \\
NFATC3 & 16 & $P L A 2 G 15$ & 16 & Intra \\
FZD2 & 10 & $R P S 24$ & 17 & Inter \\
KRT8 & 12 & $P K M 2$ & 15 & Inter \\
\hline
\end{tabular}




\section{A}
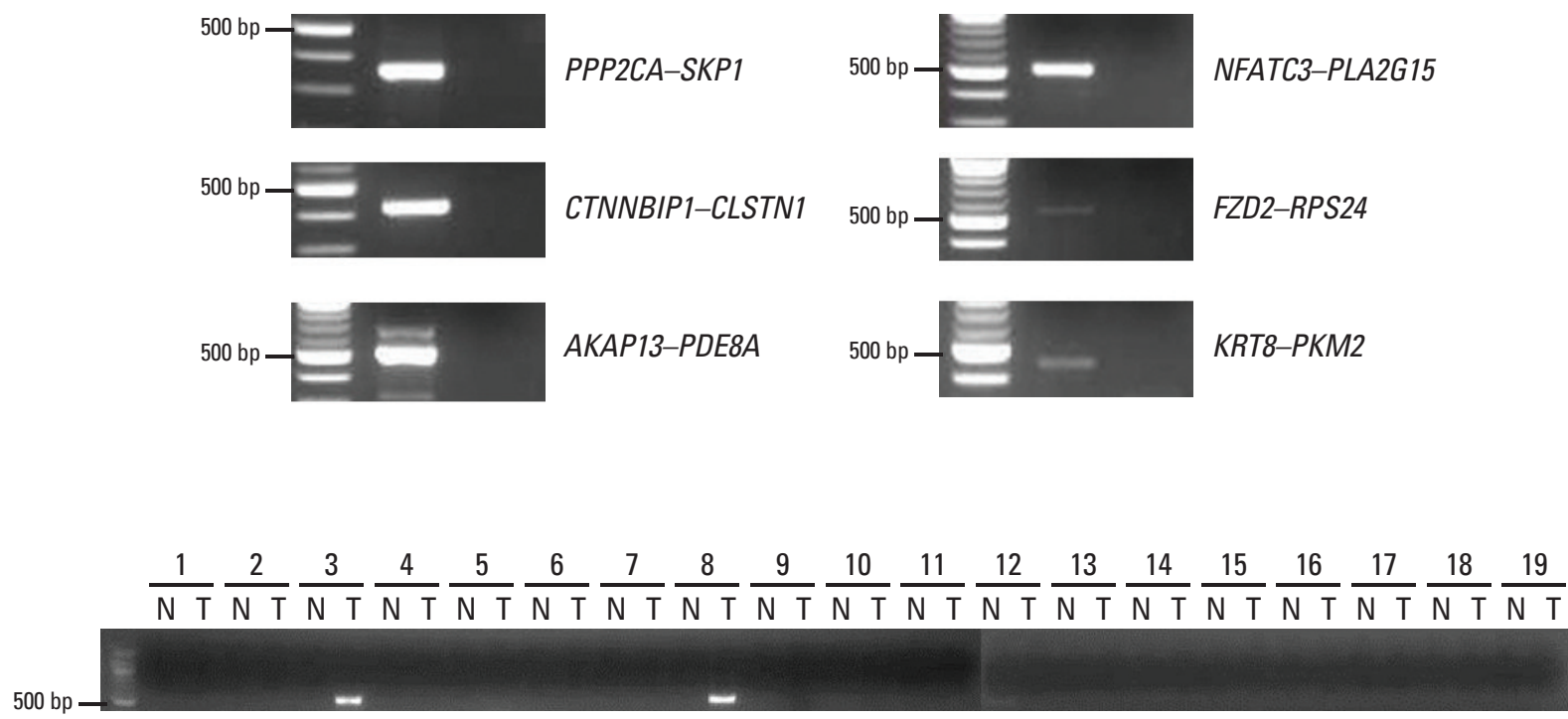

C

NFATC3

PLA2G15

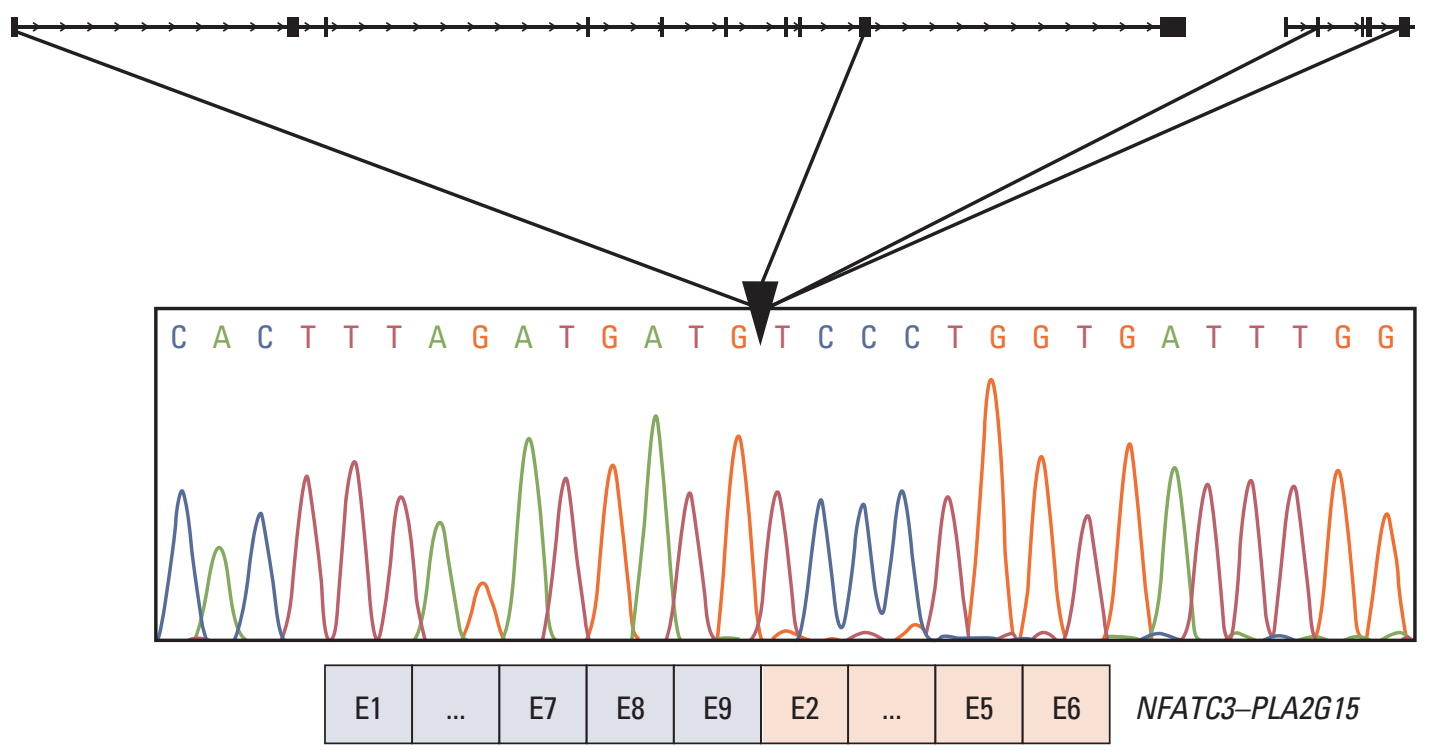

Fig. 2. Identification of 6 fusion transcripts (FTs) in the colorectal cancer (CRC) cell lines. (A) Presence of PPP2CA-SKP1, CTNNBIP1-CLSTN1, AKAP13-PDE8A, NFATC3-PLA2G15, FZD2-RPS24, and KRT8-PKM2 FTs was confirmed by reverse transcriptase-polymerase chain reaction (RT-PCR) in CRC cell lines. (B) The NFATC3-PLA2G15 FT was detected in colon cancer tissues by RT-PCR. N, normal tissue; T, tumor tissue. (C) The NFATC3-PLA2G15 FT was validated in the colo-320 cell line using Sanger-sequencing. The FT breakpoint was passed from exon 9 of NFATC3 to exon 2 of PLA2G15. (Continued to the next page) 

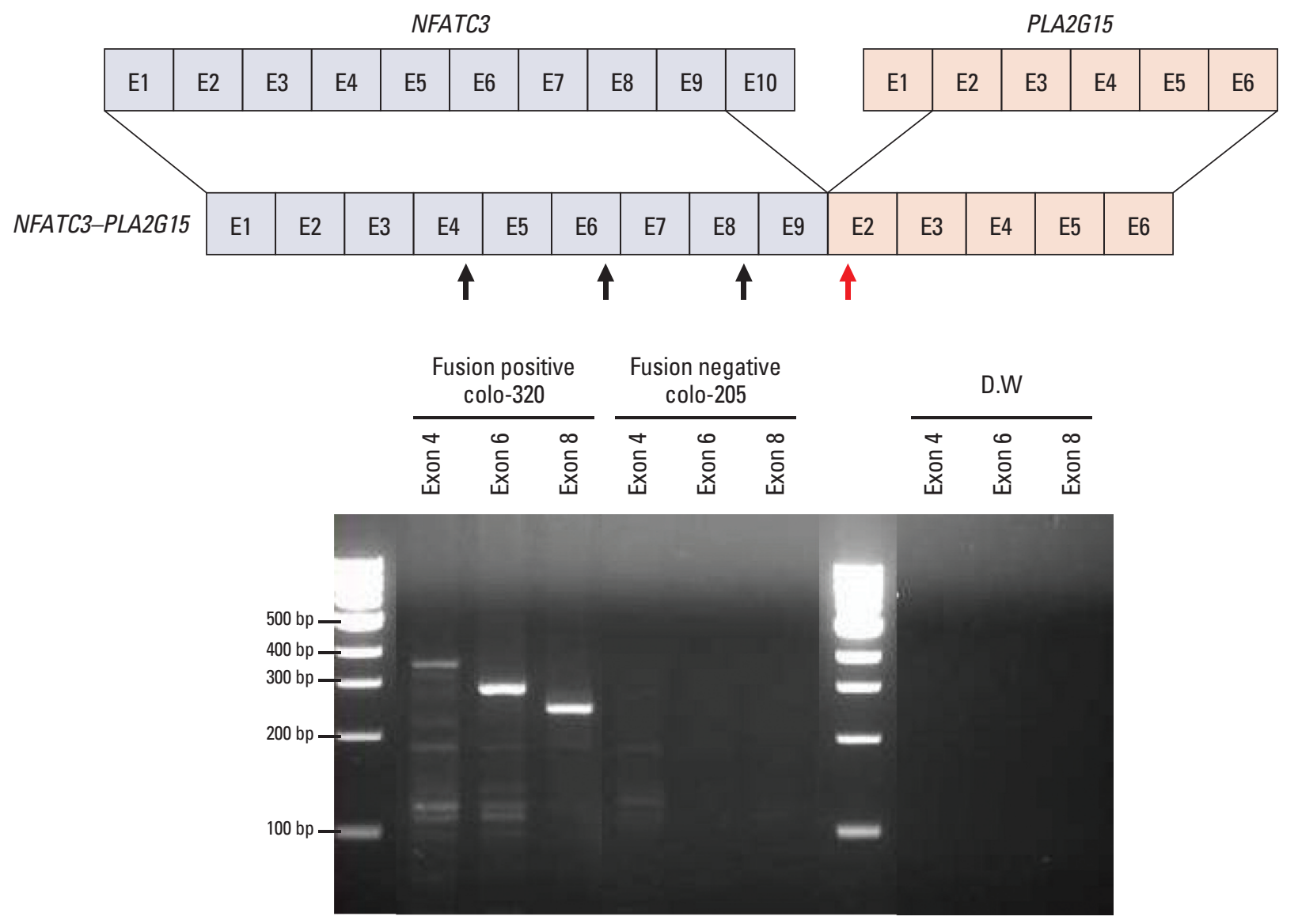

Fig. 2. (Continued from the previous page) (D) NFATC3-PLA2G15 FT mRNA expression was observed in the colo-320 fusionpositive cell line and the colo-205 fusion-negative cell line by RT-PCR. Forward primers targeted NFATC3 exon 4, 6, and 8 (black arrows) and reverse primers targeted PLA2G15 exon 2 (red arrow). Distilled water (DW) was used for the negative control.

NFATC3 and exons 2-6 of PLA2G15; fusion junctions of each transcript were confirmed by Sanger sequencing (Fig. 2C). However, no genomic-level changes were detected by longrange PCR (data not shown). We designed a set of forward PCR primers that targeted NFATC 3 exons 4,6 , and 8 , and reverse primers for PLA2G15 exon 2 to detect the FT directly by reverse transcriptase-polymerase chain reaction (Fig. 2D). We confirmed the presence of NFATC3-PLA2G15 FT in colo320 cells, but not in the fusion-negative colo- 205 cells.

\section{NFATC3-PLA2G15 FT regulates EMT}

To investigate the functional consequences of inhibiting the NFATC3-PLA2G15 FT, we designed a customized siRNA to target the fusion junction site for a knock-down NFATC3PLA2G15 FT (NFATC3-PLA2G15-KO). Reportedly, NFATC promotes epithelial-mesenchymal transition (EMT) through upregulation of Src expression in mouse embryonic stem cells [19], whereas silencing NFATC inhibited transforming growth factor $\beta$-mediated EMT [20]. Vimentin and E-cadherin are well characterized and have specific roles in mesenchymal and epithelial cellular states, respectively. We therefore screened mRNA expression levels of vimentin and E-cadherin in 28 CRC cell lines using qRT-PCR. We discovered that vimentin mRNA expression levels were the highest and E-cadherin levels were the lowest in the colo-320 cell line that harbored the NFATC3-PLA2G15 FT compared with the other 27 CRC cell lines (data not shown). Accordingly, we hypothesized that NFATC3-PLA2G15 could regulate EMTrelated genes. Hence, we used qRT-PCR to investigate mRNA expression of epithelial and mesenchymal markers after siRNA treatments. Following treatment with the NFATC3- 
A

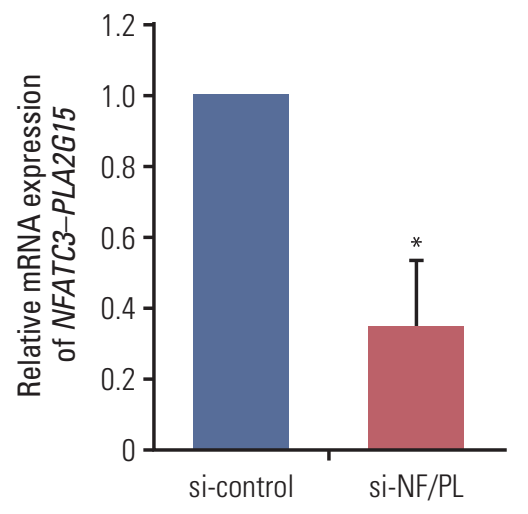

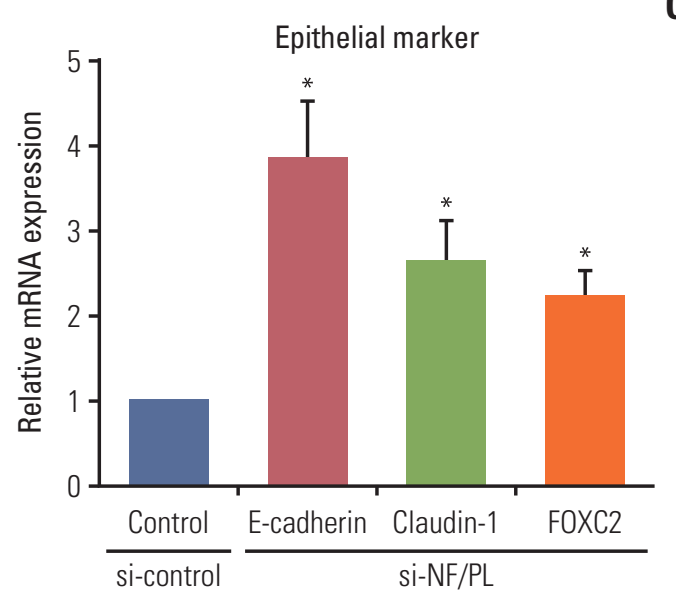

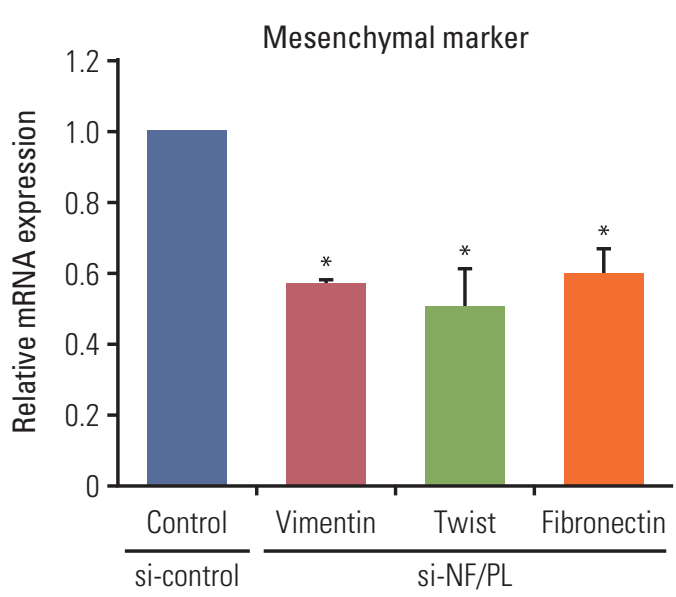

C

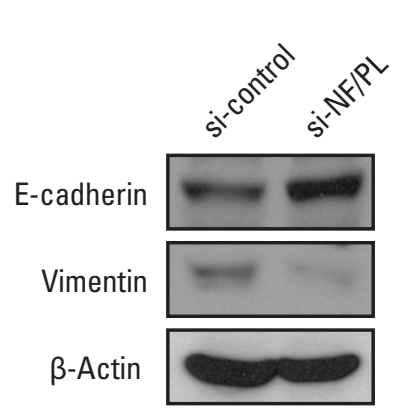

D

Fig. 3. The NFATC3-PLA2G15 fusion transcript is involved in regulation of epithelial-mesenchymal transition. (A) The colo320 cell line was transfected with NFATC3-PLA2G15 fusion transcript siRNA for 48 hours. (B, C) mRNA expression of mesenchymal markers (vimentin, twist, and fibronectin) and epithelial markers (E-cadherin, claudin-1, and FOXC2) were detected by quantitative real-time polymerase chain reaction $\left({ }^{*} \mathrm{p}<0.05\right)$. (D) Western blot analysis revealed E-cadherin, vimentin, and $\beta$-actin protein expression in the colo-320 cell line after treatment with the NFATC3-PLA2G15 fusion siRNA for 48 hours.

PLA2G15-specific siRNAs (Fig. 3A), inhibition of the FT expression also resulted in decreased mesenchymal markers-specifically, vimentin, twist, and fibronectin (Fig. 3B); and increased epithelial markers-specifically, E-cadherin, claudin-1, and FOXC2 (Fig. 3C). Western blot showed vimentin expression was decreased, and E-cadherin expression increased, in siRNA-treated cells (Fig. 3D). This result suggests that the NFATC3-PLA2G15 regulates EMT- and MET-related genes.

\section{NFATC3-PLA2G15-KO reduced cell invasion, colony for- mation, and cell proliferation}

As we observed that NFATC3-PLA2G15 FT could regulate the EMT-related genes, we investigated how NFATC3-PLA2G15-KO affected invasion ability in the cell lines. A Matrigel invasion assay demonstrated that the number of invading cells, NFATC3-PLA2G15-KO cells, was decreased compared with control cells (Fig. 4A and B).

We performed a soft agar assay to investigating the effect of NFATC3-PLA2G15-KO on colony-formation ability in the cell lines. Using fusion junction-specific siRNA, we con- 
A
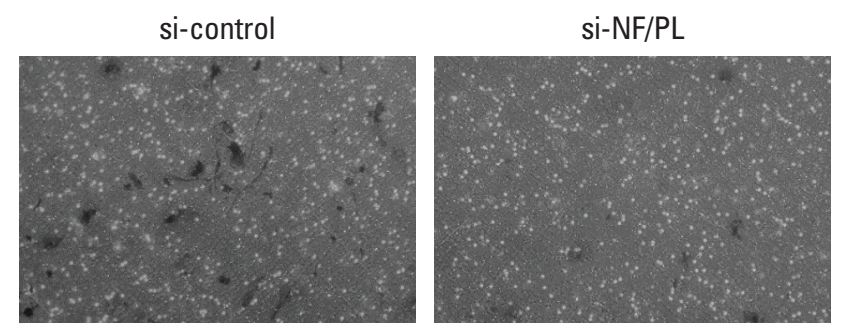
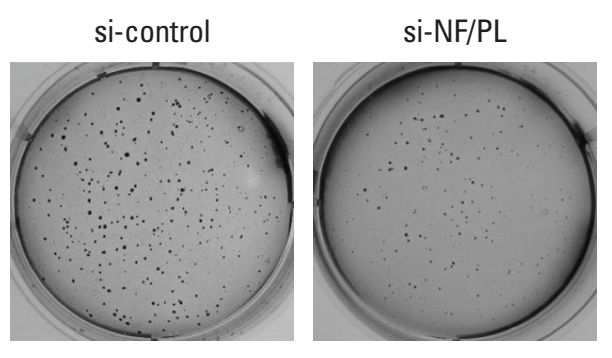

C

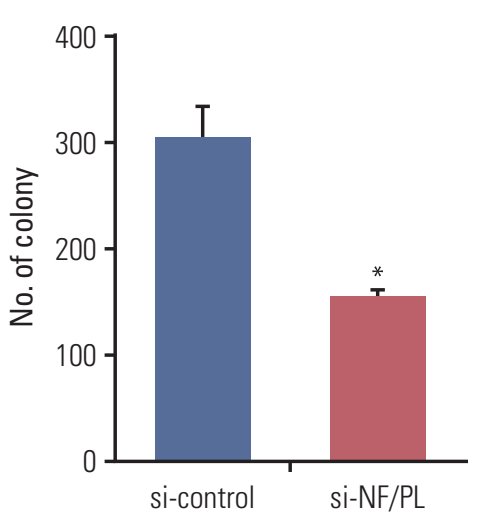

Fig. 4. The NFATC3-PLA2G15 fusion transcript affects invasiveness and has anchorage-independent abilities. (A, B) We conducted a 48-hour Matrigel invasion assay with colo-320 cells that were stably transfected with NFATC3-PLA2G15 siRNA and control cells treated with siRNA control $\left({ }^{*} \mathrm{p}<0.05\right)$. (C, D) We conducted a 2-week soft-agar independentanchorage assay on siRNA-treated colo-320 fusion-positive cell line and control cells treated with siRNA control ( $\left.{ }^{*} \mathrm{p}<0.05\right)$.

firmed knockdown of NFATC3-PLA2G15 mRNA by qRTPCR. We then observed that colony-formation was suppressed in NFATC3-PLA2G15-KO cells compared with cells treated with control siRNA (Fig. $4 \mathrm{C}$ and D). This result showed that NFATC3-PLA2G15 affected cell invasion and colony formation abilities.

We also conducted a cell-counting assay to investigate the correlation between the NFATC3-PLA2G15 and cell proliferation after siRNA treatment in the colo-320 fusion-positive and SNU-1033 fusion-negative cell lines.

During treatment with NFATC3-PLA2G15 siRNA, confluence of colo-320 cells at 48 hours after siRNA transfection was decreased (Fig. 5A and B). After siRNA treatment, proliferation was inhibited in the colo-320 cell line that had expressed NFATC3-PLA2G15 (Fig. 5C); however, no effects were observed in the SNU-1033 cell line (Fig. 5E). mRNA

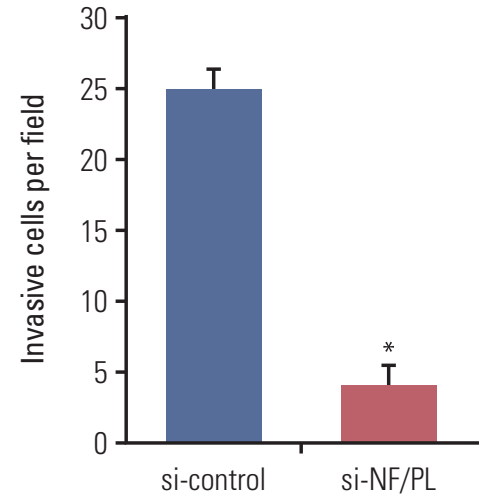

D

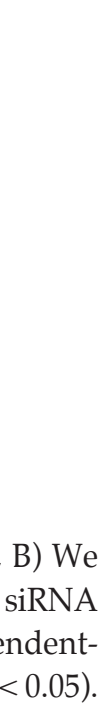




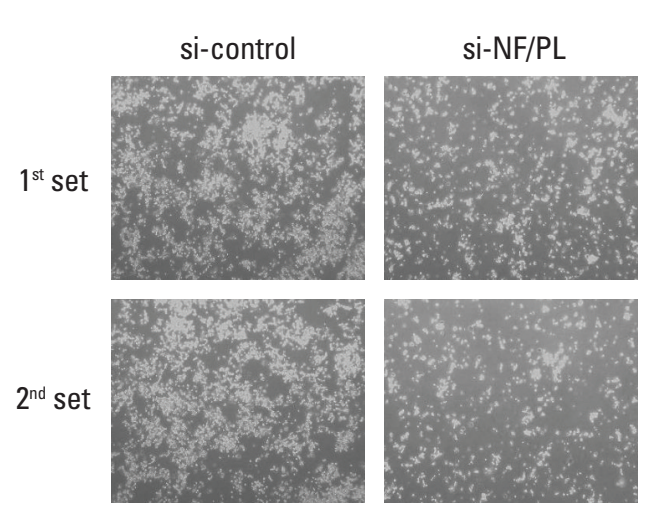

A

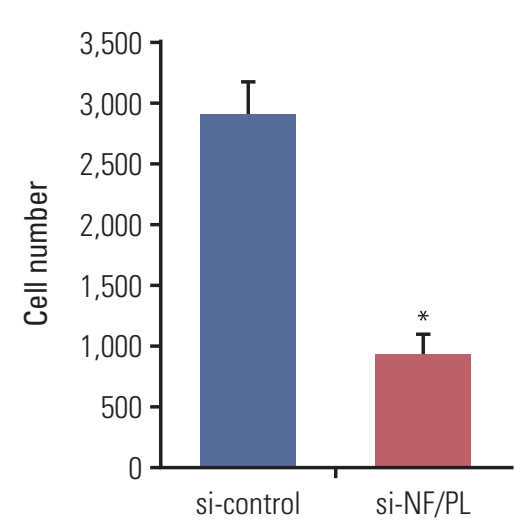

B

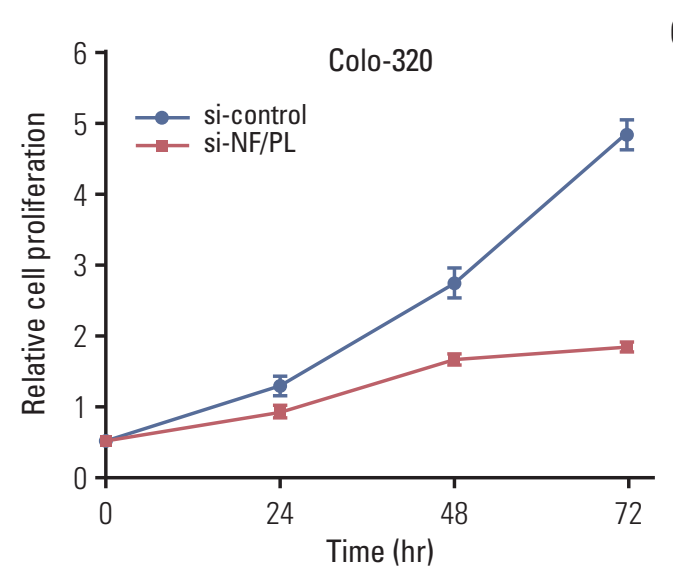

C
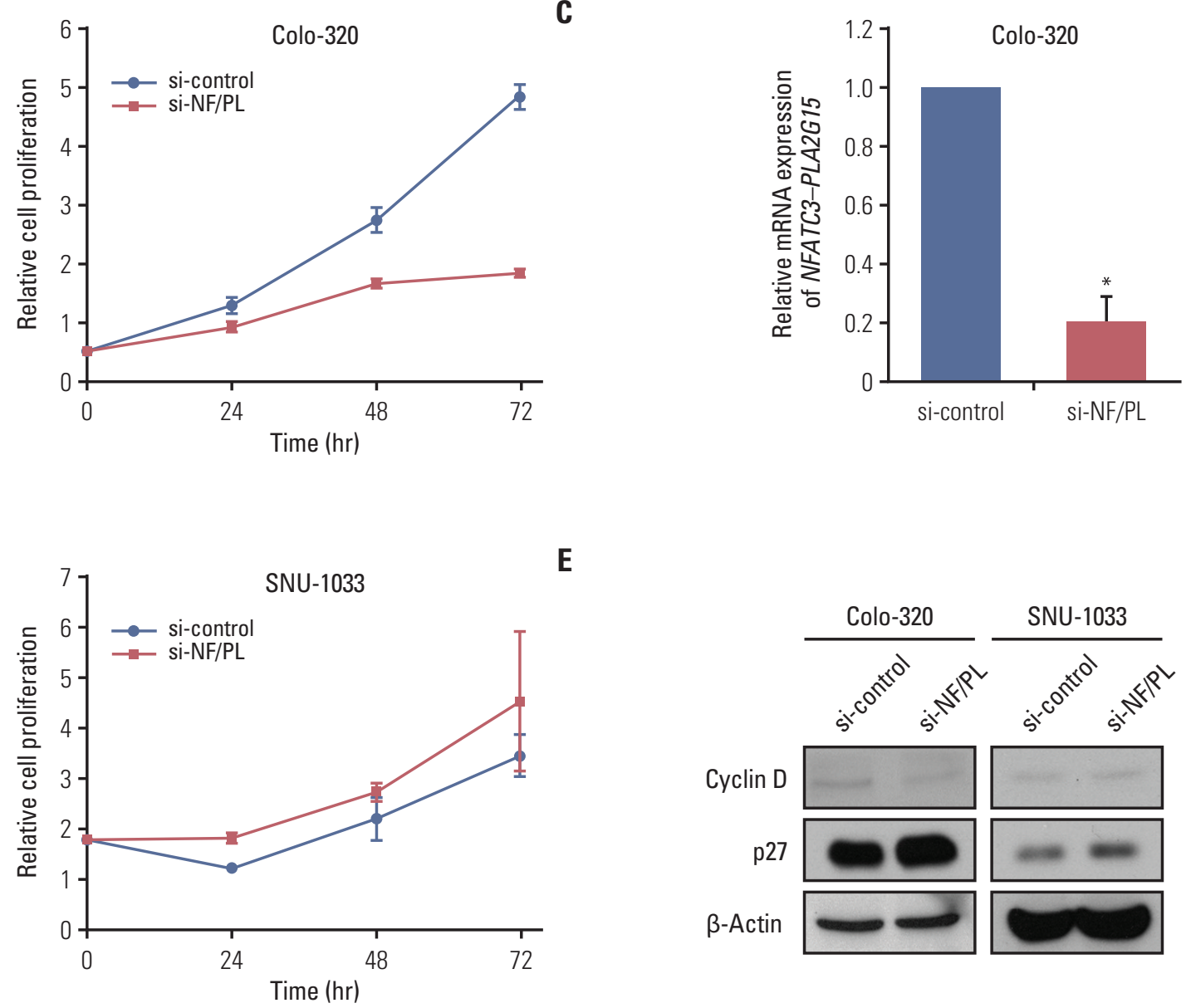

E

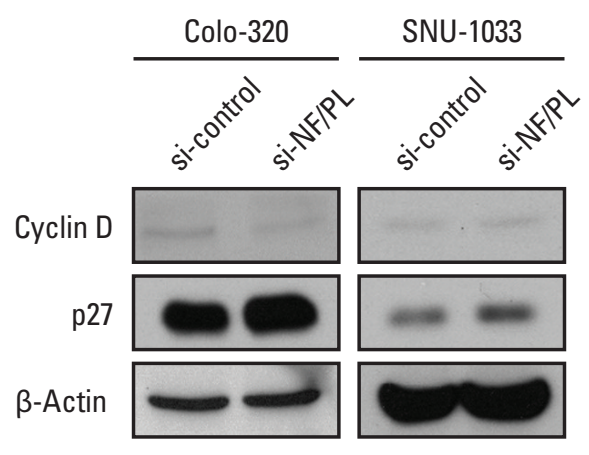

Fig. 5. The NFATC3-PLA2G15 fusion transcript effected the regulation of cell number. (A, B) The NFATC3-PLA2G15 fusion transcript siRNA was transfected into the colo-320 cell line and cell confluence was observed at 48 hours. Cells were observed by microscopy after siRNA treatment in the colo-320 fusion positive cell line. (C, E) Cell counting assay. A cell counting assay was conducted after NFATC3-PLA2G15 fusion siRNA treatment in the colo-320 fusion positive cell line and SNU-1033 fusion negative cell line in a time-dependent manner. Viable cells were counted every 3 days $\left({ }^{*} p<0.05\right)$. (D) The colo-320 cell line was transfected with NFATC3-PLA2G15 fusion transcript siRNA for 48 hours. (F) Western-blotting analysis revealed cyclin D, and p27 protein expression in the colo-320 and SNU-1033 cell lines after treatment with the NFATC3-PLA2G15 fusion siRNA for 48 hours. 
filtered them for in-frame fusion patterns to obtain six fusion candidates (PPP2CA-SKP1, CTNNBIP1-CLSTN1, AKAP13PDE8A, NFATC3-PLA2G15, FZD2-RPS24, and KRT8-PKM2). Among them, we focused on the NFATC3-PLA2G15 FT because it showed tumor-specific characteristics. NFATC3PLA2G15 FT has previously been reported in a normal-karyotype AML having the same fusion junction as we discovered in this study [21]. However, this study is the first to conduct a functional study of this FT. Although fusion break point is different compared with NFATC3-PLA2G15 FT in our paper, it was additionally detected in T-acute lymphoblastic leukemia recently [22]. The transcription factor, nuclear factor of activated T-cells (NFAT), exists as five isoforms (NFAT1, NFAT2, NFAT3, NFAT4, and NFAT5 [23], and is known to be involved in the non-canonical WNT signaling pathway of the immune system and the WNT/calcium pathway [24]. The binding of $\mathrm{Ca}^{+}$to calmodulin activates calcineurin, which dephosphorylates and activates the NFAT transcription factors. Dephosphorylated NFAT then translocates to the nucleus where it interacts with diverse transcriptional partners and regulates the transcription of target genes through a nuclear localization signal (NLS) peptide [25]. NFAT is then re-phosphorylated and inactivated by NFAT kinases, including GSK3, CK1, and DYRK1, after which NFAT translocates back to the cytosol through a nuclear export signal (NES) peptide [26]. The phospholipase A2 Group XV (PLA2G15) protein (also known as lysosomal phospholipase A2 [LPLA2]) acts on the membrane to regulate lipid catabolism; it has calcium-independent activity [27]. Phospholipase mediates of intra- and extra-cellular signaling and affects various tumorigenic processes, including cell migration, invasion, proliferation and angiogenesis [28].

We found that NFATC3-PLA2G15 FT includes exon 1-9 of NFATC3 and exon 2-6 of PLA2G15. Thus, this FT has the NFATC3 NLS peptide like NFATC3 full-length gene, but the NES peptide was deleted when the transcript fuses with PLA2G15. In accordance with it, we hypothesized that the NFATC3-PLA2G15 FT could not translocate back to the cytosol because it did not have the complete NES signal peptide. We tried to investigate this mechanism, but failed to make FT construct and FT gene synthesis. Therefore, follow-up study is needed to discover the mechanism. In accord with a prior report indicating that NFATC3 is involved in the regulation of cell proliferation, we observed that the NFATC3PLA2G15 FT regulated cell proliferation as a driver gene despite the fact we could not reveal the functional mechanisms. Another group observed that NFAT blocker A-285222 inhibited cell proliferation following a reduction in cyclin D expression [29]. Although we did not investigate a relationship between the NFATC3-PLA2G15 FT and any drug, it could potentially serve as a therapeutic target in CRC. In other reports, the fusion genes containing druggable tyrosine kinase (ALK, FGFR2, KIT, NTRK1, NTRK2, RET and PDG$F R A$ ) was verified in colorectal cancer cell lines using targeted approach [30]. As this case, the other 5 fusion candidates from our study are also potential drug targets, and should be screened with more paired tumor tissues. Other than 6 FTs identified through targeted gene filtering step, the functional study of the other high-frequency FTs from other filtering methods, will be needed.

In summary, we have identified expression of NFATC3PLA2G15 FT in CRC cell lines using RNA sequencing, and demonstrated that recurrent in-frame NFATC3-PLA2G15 FT promoted tumor cell proliferation and invasion ability in CRC cell lines.

\section{Electronic Supplementary Material}

Supplementary materials are available at Cancer Research and Treatment website (https:// www.e-crt.org).

\section{Conflicts of Interest}

Conflict of interest relevant to this article was not reported.

\section{Acknowledgments}

This research was supported by a grant from the Korea Health Technology R\&D Project through the Korea Health Industry Development Institute the Ministry of Health \& Welfare (HI14C1277 and 2013R 1A 2058552).

\section{References}

1. Siegel R, Desantis C, Jemal A. Colorectal cancer statistics, 2014. CA Cancer J Clin. 2014;64:104-17.

2. Allen TM. Ligand-targeted therapeutics in anticancer therapy. Nat Rev Cancer. 2002;2:750-63.

3. Maher CA, Palanisamy N, Brenner JC, Cao X, Kalyana-Sun- daram S, Luo S, et al. Chimeric transcript discovery by pairedend transcriptome sequencing. Proc Natl Acad Sci U S A. 2009;106:12353-8.

4. Maher CA, Kumar-Sinha C, Cao X, Kalyana-Sundaram S, Han $B$, Jing $X$, et al. Transcriptome sequencing to detect gene 
fusions in cancer. Nature. 2009;458:97-101.

5. Ozsolak F, Milos PM. RNA sequencing: advances, challenges and opportunities. Nat Rev Genet. 2011;12:87-98.

6. Kim M, Lee KH, Yoon SW, Kim BS, Chun J, Yi H. Analytical tools and databases for metagenomics in the next-generation sequencing era. Genomics Inform. 2013;11:102-13.

7. Griswold IJ, MacPartlin M, Bumm T, Goss VL, O'Hare T, Lee $\mathrm{KA}$, et al. Kinase domain mutants of Bcr-Abl exhibit altered transformation potency, kinase activity, and substrate utilization, irrespective of sensitivity to imatinib. Mol Cell Biol. 2006;26:6082-93.

8. Bunting SF, Nussenzweig A. End-joining, translocations and cancer. Nat Rev Cancer. 2013;13:443-54.

9. Mitelman F, Johansson B, Mertens F. The impact of translocations and gene fusions on cancer causation. Nat Rev Cancer. 2007;7:233-45.

10. Shaw AT, Hsu PP, Awad MM, Engelman JA. Tyrosine kinase gene rearrangements in epithelial malignancies. Nat Rev Cancer. 2013;13:772-87.

11. Soda M, Choi YL, Enomoto M, Takada S, Yamashita Y, Ishikawa S, et al. Identification of the transforming EML4-ALK fusion gene in non-small-cell lung cancer. Nature. 2007;448: 561-6.

12. Antoniu SA. Crizotinib for EML4-ALK positive lung adenocarcinoma: a hope for the advanced disease? Evaluation of Kwak EL, Bang YJ, Camidge DR, et al. Anaplastic lymphoma kinase inhibition in non-small-cell lung cancer. N Engl J Med 2010;363(18):1693-703. Expert Opin Ther Targets. 2011;15: 351-3.

13. Bass AJ, Lawrence MS, Brace LE, Ramos AH, Drier Y, Cibulskis K, et al. Genomic sequencing of colorectal adenocarcinomas identifies a recurrent VTI1A-TCF7L2 fusion. Nat Genet. 2011;43:964-8.

14. Seshagiri S, Stawiski EW, Durinck S, Modrusan Z, Storm EE, Conboy CB, et al. Recurrent R-spondin fusions in colon cancer. Nature. 2012;488:660-4.

15. Nome T, Hoff AM, Bakken AC, Rognum TO, Nesbakken A, Skotheim RI. High frequency of fusion transcripts involving TCF7L2 in colorectal cancer: novel fusion partner and splice variants. PLoS One. 2014;9:e91264.

16. Nome T, Thomassen GO, Bruun J, Ahlquist T, Bakken AC, Hoff AM, et al. Common fusion transcripts identified in colorectal cancer cell lines by high-throughput RNA sequencing. Transl Oncol. 2013;6:546-53.

17. Lovf M, Nome T, Bruun J, Eknaes M, Bakken AC, Mpindi JP, et al. A novel transcript, VNN1-AB, as a biomarker for colorectal cancer. Int J Cancer. 2014;135:2077-84.
18. Ku JL, Park JG. Biology of SNU cell lines. Cancer Res Treat. 2005;37:1-19.

19. Li X, Zhu L, Yang A, Lin J, Tang F, Jin S, et al. CalcineurinNFAT signaling critically regulates early lineage specification in mouse embryonic stem cells and embryos. Cell Stem Cell. 2011;8:46-58.

20. Sengupta S, Jana S, Biswas S, Mandal PK, Bhattacharyya A. Cooperative involvement of NFAT and SnoN mediates transforming growth factor-beta (TGF-beta) induced EMT in metastatic breast cancer (MDA-MB 231) cells. Clin Exp Metastasis. 2013;30:1019-31.

21. Wen H, Li Y, Malek SN, Kim YC, Xu J, Chen P, et al. New fusion transcripts identified in normal karyotype acute myeloid leukemia. PLoS One. 2012;7:e51203.

22. Bond J, Tran Quang C, Hypolite G, Belhocine M, Bergon A, Cordonnier $\mathrm{G}$, et al. Novel intergenically spliced chimera, NFATC3-PLA2G15, is associated with aggressive T-ALL biology and outcome. Mol Cancer Res. 2018;16:470-5.

23. Hoey T, Sun YL, Williamson $K$, Xu X. Isolation of two new members of the NF-AT gene family and functional characterization of the NF-AT proteins. Immunity. 1995;2:461-72.

24. Oh-hora M, Rao A. The calcium/NFAT pathway: role in development and function of regulatory $\mathrm{T}$ cells. Microbes Infect. 2009;11:612-9.

25. Branden LJ, Mohamed AJ, Smith CI. A peptide nucleic acidnuclear localization signal fusion that mediates nuclear transport of DNA. Nat Biotechnol. 1999;17:784-7.

26. MacDonnell SM, Weisser-Thomas J, Kubo H, Hanscome M, Liu Q, Jaleel N, et al. CaMKII negatively regulates calcineurinNFAT signaling in cardiac myocytes. Circ Res. 2009;105: 316-25.

27. Abe A, Kelly R, Shayman JA. The measurement of lysosomal phospholipase A2 activity in plasma. J Lipid Res. 2010;51:246470.

28. Park JB, Lee CS, Jang JH, Ghim J, Kim YJ, You S, et al. Phospholipase signalling networks in cancer. Nat Rev Cancer. 2012;12:782-92.

29. Nilsson LM, Sun ZW, Nilsson J, Nordstrom I, Chen YW, Molkentin JD, et al. Novel blocker of NFAT activation inhibits IL-6 production in human myometrial arteries and reduces vascular smooth muscle cell proliferation. Am J Physiol Cell Physiol. 2007;292:C1167-78.

30. Medico E, Russo M, Picco G, Cancelliere C, Valtorta E, Corti $\mathrm{G}$, et al. The molecular landscape of colorectal cancer cell lines unveils clinically actionable kinase targets. Nat Commun. 2015;6:7002. 